\title{
MHD oscillatory Couette flow of a radiating viscous fluid in a porous medium with periodic wall temperature
}

\author{
C. Israel - Cookey ${ }^{+}$, E. Amos, C. Nwaigwe \\ Department of Mathematics \\ Rivers State University of Science and Technology, Port Harcourt 500001, Nigeria. \\ ${ }^{+}$Email: israel-cookey.chigozie@ust.edu.ng; cookeyci@yahoo.com.
}

\begin{abstract}
This paper investigates the problem of MHD free convection and oscillatory flow of an optically thin fluid bounded by two horizontal porous parallel walls under the influence of an external imposed transverse magnetic field in a porous medium. By taking the radiative heat flux in the differential form and imposing an oscillatory time-dependent perturbation the coupled nonlinear problem is solved for the velocity and temperature profiles. The results show that the fluid velocity increased with increase in the magnetic field, $M$, Grashof number, Gr, and the porosity, $\chi$ parameters; whereas the fluid velocity is decreased with increase in the radiation parameter, $F$. Equally, the skin friction decreased with increases in magnetic and porosity parameters for $G r=2$ and increased for $G r=5$, while the reverse is the case with increase in radiation. Finally, the rate of heat transfer is increased with increases in the radiation parameter.
\end{abstract}

Keywords: MHD, Oscillatory, Couette flow, optically thin fluid, porous medium.

\section{INTRODUCTION}

The phenomenon of free or natural convection arises in fluids when temperature changes cause density variations leading to buoyancy forces acting on the fluid particles. Such flows which are driven by temperature differences abound in nature and have been studied extensively because of its applications in engineering, geophysical and astrophysical environments. Comprehensive literature on various aspects of free convection flows and its applications could be found in Ghoshdastidar (2004), Nield and Bejan (2006). In particular Ghoshdastidar (2004) gave various areas of applications of free convection flow such as those found in heat transfer from pipes and transmission lines as well as from electronic devices, heat dissipation from the coil of a refrigerator unit to the surrounding air, heat transfer from a heater to room air, heat transfer in nuclear fuel rods to the surrounding coolant, heated and cooled enclosures, quenching, wire - drawing and extrusion, atmospheric and oceanic circulation.

Buoyancy - driven flows over porous materials enhances heat transfer. These are encountered in a wide range of thermal engineering applications such as in geothermal systems, oil extraction; ground water pollution, thermal insulation, heat exchangers, storage of nuclear wastes, packed bed catalytic reactors. Most of these application exhibit high temperature phenomena such as those found in astrophysical environments, solar power technology, MHD power generation, space reentry vehicles nuclear engineering (Bestman and Adjepong (1988), Israel - Cookey et al. (2003)). In this case, the effect of radiative heat flux cannot be ignored.

An analysis of flow of Couette flow has been studied extensively for the case of horizontal channel. Choi et al. (1994) studied the buoyancy effects in plane Couette flow heated uniformly from below with constant heat flux. Alagoa et al. (1999) studied the problem of radiative and free convection effects of a flow through porous medium between two infinite parallel plates with time - dependent suction. Attia and Sayed - Ahmed (2004) investigated the problem of the effect Hall currents on unsteady MHD Couette flow and heat transfer of a Bingham fluid with suction and injection. Makinde and Osalusi (2006) considered the problem of MHD steady flow in a channel filled with porous material with slip at the boundaries; while Mebine (2007) studied the effect of thermal radiation on MHD Couette flow with heat transfer between two parallel plates.

In this present paper, we investigate the problem of MHD oscillatory Couette flow of a radiating viscous fluid with period wall temperatures in a porous 
medium when lower plate is suddenly set in motion with the upper plate stationary.

Mathematical Formulation: We consider the unsteady Couette flow of an electrically conducting and optically thin viscous incompressible fluid in a porous medium bounded by two infinite non conducting horizontal parallel walls under the influence of an externally applied uniform magnetic field $\mathbf{B}_{0}$ and radiative heat flux. It is assumed that the fluid has small electrical conductivity and the electromagnetic force produced is very small, so we neglect the induced magnetic field. The lower wall is suddenly moved from rest with a free stream velocity, $u_{m}^{*}$ that oscillates in time about a constant mean velocity, $U_{0}$. Further, it is assumed that the temperature of the moving lower plate oscillates in time about a non - zero constant mean.

Then, assuming Boussinesq approximation for an incompressible fluid model, the governing equations of the flow for an optically thin medium are

$$
\begin{gathered}
\rho \frac{\partial u^{*}}{\partial t^{*}}=\rho \frac{d U^{*}}{d t^{*}}+\mu \frac{\partial^{2} u^{*}}{\partial y^{* 2}}+\rho g \beta\left(T^{*}-T_{s}^{*}\right)-\left(\frac{1}{k^{*}}+\sigma_{c} B_{0}^{2}\right)\left(u^{*}-U^{*}\right) \\
\frac{\partial T^{*}}{\partial t^{*}}=\frac{\kappa}{\rho c_{p}} \frac{\partial^{2} T^{*}}{\partial y^{* 2}}-\frac{1}{\rho c_{p}} \frac{\partial q}{\partial y^{*}}
\end{gathered}
$$

with the following boundary conditions

$$
\begin{gathered}
u^{*}=U_{0}\left(1+\varepsilon e^{i \omega^{*} t}\right), T^{*}=T_{m}^{*}+\varepsilon\left(T_{m}^{*}-T_{s}^{*}\right) e^{i \omega^{*} t} \\
\text { on } y^{*}=0 \quad \text { (3a) } \\
u^{*}=0, T^{*}=T_{s}^{*} \\
\text { on } y^{*}=h \quad \text { (3b) }
\end{gathered}
$$

where

$$
u^{*}, U^{*}, U_{0}, \mu, \rho, g, \beta, \sigma_{\mathrm{c}}, k^{*}, \kappa, c_{p}, T^{*}, T_{s}, T_{m}, q
$$

are respectively velocity, free stream velocity, mean constant free stream velocity, viscosity, density, gravitational acceleration, coefficient of thermal expansion, electric conductivity of the fluid, permeability of the porous medium, thermal conductivity, specific heat capacity, temperature of the fluid, temperature of the upper wall, temperature of the lower wall and the radiative heat flux. Also, $\omega^{*}$ is the frequency of oscillation, $\varepsilon<<1$ a small parameter and $U^{*}\left(t^{*}\right)=1+\varepsilon e^{i \omega^{*} t^{*}}$ is the free stream velocity.

We assume that the wall temperatures, $T_{m}$ and $T_{s}$ are high enough to induce radiative heat transfer. Since the fluid is optically thin with relatively low density, then in the spirit of Cogley et al. (1968) the radiative heat flux, $q$ in the energy equation (2) is taken as

$$
\frac{\partial q}{\partial y}=4 \alpha^{2}\left(T^{*}-T_{s}\right)
$$

where $\alpha$ is the radiation absorption coefficient. Using Equation (4) in (2), we then rewrite the governing equations in dimensionless form as

$\omega \frac{\partial u}{\partial t}=\omega \frac{\partial U}{\partial t}+\frac{\partial^{2} u}{\partial y^{2}}+G r \theta-\left(\chi^{2}+M^{2}\right)(u-U)$

$$
\omega \operatorname{Pr} \frac{\partial \theta}{\partial t}=\frac{\partial^{2} \theta}{\partial y^{2}}-F^{2} \theta
$$

and

$$
U(t)=1+\varepsilon e^{i t}
$$

with the corresponding boundary conditions

$$
\begin{gathered}
u=1+\varepsilon e^{i t}, \theta=1+\varepsilon e^{i t} \\
\text { on } y=0 \\
u=0, \theta=0 \\
\text { on } y=1
\end{gathered}
$$

In writing the above dimensionless equations, we have used the following dimensionless variables and parameters

$$
\begin{aligned}
& y=\frac{y^{*}}{h}, u=\frac{u^{*}}{U_{0}}, U=\frac{U^{*}}{U_{0}}, t=\omega^{*} t^{*}, \omega=\frac{\omega^{*} h^{2}}{v}, \theta=\frac{\left(T^{*}-T_{s}\right)}{\left(T_{m}-T_{s}\right)}, \chi^{2}=\frac{h^{2}}{k^{*}}, \\
& M^{2}=\frac{\sigma_{c} B h^{2}}{\rho v}, \operatorname{Pr}=\frac{v}{\alpha^{*}}, \alpha^{*}=\frac{\kappa}{\rho_{p}}, G r=\frac{g h^{2}\left(T_{m}-T_{s}\right)}{U_{0}}, F^{2}=\frac{4 \alpha^{2} h^{2}}{\kappa}
\end{aligned}
$$

$U(t)=1+\mathscr{Q}^{i t}$

where $G r$ is the Grashof number, $M$ is the magnetic parameter, $F$ is the radiation parameter.

\section{Method of Solution}

The problem posed in Equations (5) - (8) are highly coupled partial differential equations in the flow 
variables and cannot be solved exactly. But, since $\varepsilon<<1$, we can advance solutions for the flow velocity and temperature using the regular perturbation of the form:

$$
\begin{aligned}
& u(y, t)=u_{0}(y)+\varepsilon u_{1}(y) e^{i t}+O\left(\varepsilon^{2}\right) \\
& \theta(y, t)=\theta_{0}(y)+\varepsilon \theta_{1}(y) e^{i t}+O\left(\varepsilon^{2}\right)
\end{aligned}
$$

Substituting Equation (9) into Equations (5) - (8), neglecting the coefficients of $O\left(\varepsilon^{2}\right)$ and simplifying, we obtain the following sequence of approximations

$$
\begin{gathered}
u_{0}^{\prime \prime}-\left(\chi^{2}+M^{2}\right) u_{0}=-\left(\chi^{2}+M^{2}\right)-G r \theta_{0} \\
\theta_{0}^{\prime \prime}-F^{2} \theta_{0}=0
\end{gathered}
$$

subject

$$
\begin{array}{ll}
u_{0}=1, \theta_{0}=1 & \text { on } y=0 \\
u_{0}=0, \theta_{0}=0 & \text { on } y=1
\end{array}
$$

for $O(1)$ equations, and

$u_{1}^{\prime \prime}-\left(\chi^{2}+M^{2}+i \omega\right) u_{1}=-\left(\chi^{2}+M^{2}+i \omega\right)-G r \theta_{1}$

$$
\theta_{1}^{\prime \prime}-\left(F^{2}+i \omega\right) \theta_{1}=0
$$

subject to

$$
\begin{array}{ll}
u_{1}=1, \theta_{1}=1 & \text { on } y=0 \\
u_{1}=0, \theta_{1}=0 & \text { on } y=1
\end{array}
$$

Solving Equations (11) - (12) with boundary conditions (13) and substituting the results in to Equations (14) - (15) and solving the resulting equations using boundary conditions (16), we obtain the velocity and temperature profiles as

$$
\begin{aligned}
u(y, t)= & \frac{1}{\left(F^{2}-\lambda^{2}\right)\left(1-e^{2 \lambda}\right)}\left(e ^ { - y ( 2 F + \lambda ) } \left(e^{2 F y}\left(e^{\lambda y}+e^{\lambda+2 \lambda y}-e^{(2+y) \lambda}-e^{-\lambda}\right)\left(F^{2}-\lambda^{2}\right)\right.\right. \\
+ & \frac{G r q}{\left(F^{2}-\lambda^{2}\right)\left(1-e^{2 \lambda}\right)}\left(e^{2(F+\lambda) y}-e^{(F+\lambda)(2+y)}-e^{(3 F+\lambda) y}-e^{2(\lambda+F y)}+e^{2(F+\lambda+F y)}-e^{2(F+(F+\lambda) y)}\right. \\
+ & \left.e^{(2 F+(F+\lambda) y)}+e^{2 \lambda+(3 F+\lambda) y}\right)+\frac{x^{i t} e^{-y(2 \sqrt{\delta}+\Lambda)}}{\left(\Lambda^{2}-\delta\right)\left(1-e^{2 \Lambda}\right)}\left(e^{2 \sqrt{\delta} y}\left(e^{\Lambda y}-e^{(2+y) \Lambda}+e^{(1+2 y) \Lambda}-e^{\Lambda}\right)\left(\delta-\Lambda^{2}\right)\right. \\
+ & G r a_{2}\left(e^{2 y(\sqrt{\delta}+\Lambda)}-e^{(2+y)(\sqrt{\delta}+\Lambda}-e^{y(3 \sqrt{\delta}+\Lambda)}-e^{2(y \sqrt{\delta}+\Lambda)}+e^{2((1+y) \sqrt{\delta}+\Lambda)}+e^{(2+y) \sqrt{\delta}+\lambda y}\right. \\
& \left.+e^{(3 \sqrt{\delta}+\Lambda) y+2 \Lambda}-e^{2(1+y) \sqrt{\delta}+2 \lambda y}\right)
\end{aligned}
$$

$$
\theta(y, t)=\frac{1}{a_{1}}\left(e^{F y}-e^{-F(y-2)}\right)+\frac{\varepsilon}{a_{2}}\left(e^{\delta y}-e^{-\delta(y-2)}\right) e^{i t}
$$

where

$$
\begin{array}{ll}
a_{1}=\frac{1}{1-e^{2 F},} & a_{2}=\frac{1}{1-e^{2 \sqrt{\delta}},} \\
+i \omega \operatorname{Pr}, & \lambda^{2}=\chi^{2}+M^{2},
\end{array}
$$

$\delta=F^{2}+i \omega \operatorname{Pr}$,

$\Lambda^{2}=\lambda^{2}+i \omega \operatorname{Pr}$.

From the physical point of view, it is necessary to know the skin friction or shear and the rate of heat transfer (or the Nusselt number) on the walls. The skin friction at the lower wall is given

$$
\tau=\left.\frac{\partial u}{\partial y}\right|_{y=0}
$$

while the rate of heat transfer Nusselt number) is

$$
N u=\left.\frac{\partial \theta}{\partial y}\right|_{y=0}
$$

It is emphasized here that for want of space the expressions for the skin friction (20) and the Nusselt number (21) are not given, but are discussed quantitatively for various values of the material parameters entering the fluid in the next section.

\section{RESULTS AND DISCUSSION}

The problem of MHD free convection and oscillatory flow of an optically thin fluid bounded by two parallel walls under the influence of an external imposed transverse magnetic field in a porous medium has been studied. By taking the radiative heat flux in the differential form and imposing an oscillatory timedependent perturbation the coupled nonlinear problem is solved for the velocity and temperature profiles. In order to understand the physical situation of the problem and hence the manifestations of the various material parameters entering the problem we have computed the numerical values of the velocity, temperature, skin friction and the Nusselt number using the software "mathematica". For the purposes of our computation, we set $\varepsilon=0.02, \omega=5$, $t=\pi / 2$ and the Prandtl number, $\operatorname{Pr}=0.71$ which physically corresponds to the atmospheric environment (air) at $20^{\circ} \mathrm{C}$ fixed for the velocity and temperature profiles.

In Figures 1 - 4 we depict the velocity profile (Equation 17) for various values of radiation parameter, $F$, Grashof number, $G r$, magnetic field parameter, $M$ and the porosity parameter, $\chi$. It is interesting to observe that the fluid velocity increased with increase in the magnetic field, $M$, Grashof number, $G r$, and the porosity, $\chi$ parameters; while the reverse is the case with increase in the radiation 
parameter, $F$. Similarly, the magnitudes of the skin friction for various values of the material parameters are shown in Tables $1-3$. It is observed that the skin friction decreased with increases in magnetic and porosity parameters for $\mathrm{Gr}=2$ and increased for $G r=5$; the reverse is the case with the radiation parameter.

Table 1: Skin friction for variation of magnetic field and Grashof number

\begin{tabular}{|l|l|l|}
\hline $\mathbf{M}$ & $\tau$ & $\tau$ \\
\hline 0.5 & $\mathbf{G r}=\mathbf{2}$ & $\mathbf{G r}=\mathbf{5}$ \\
1.0 & 0.427979 & 0.366666 \\
1.5 & 0.341348 & 0.420780 \\
2.0 & 0.225900 & 0.490254 \\
2.5 & 0.107657 & 0.556827 \\
\hline
\end{tabular}

Table 2: Skin friction for variation of porosity parameter and Grashof number

\begin{tabular}{|l|l|l|}
\hline & $\tau$ & $\tau$ \\
\hline 0.1 & $\mathbf{G r}=\mathbf{2}$ & $\mathbf{G r}=\mathbf{5}$ \\
0.2 & 0.109924 & 0.555610 \\
0.3 & 0.109344 & 0.555922 \\
0.4 & 0.107634 & 0.556839 \\
0.5 & 0.104814 & 0.558350 \\
0.6 & 0.100915 & 0.560430 \\
0.7 & 0.095982 & 0.563050 \\
0.8 & 0.0900704 & 0.566171 \\
0.9 & 0.0832444 & 0.569750 \\
1.0 & 0.0755768 & 0.573735 \\
& 0.0614720 & 0.578072 \\
\hline
\end{tabular}

Table 3: Skin friction for variation of radiation parameter and Grashof number

\begin{tabular}{|c|c|c|}
\hline F & $\begin{array}{l}\tau \\
\mathbf{G r}=\mathbf{2}\end{array}$ & $\begin{array}{l}\tau \\
\mathbf{G r}=\mathbf{5}\end{array}$ \\
\hline 0.5 & 0.0205725 & 0.774538 \\
\hline 1.0 & 0.0423447 & 0.720108 \\
\hline 1.5 & 0.0731078 & 0.643200 \\
\hline 2.0 & 0.1076570 & 0.556827 \\
\hline 2.5 & 0.1421260 & 0.470655 \\
\hline 3.0 & 0.1743390 & 0.390121 \\
\hline 3.5 & 0.2034090 & 0.317447 \\
\hline 4.0 & 0.2291960 & 0.252980 \\
\hline
\end{tabular}

$\mathrm{u}$

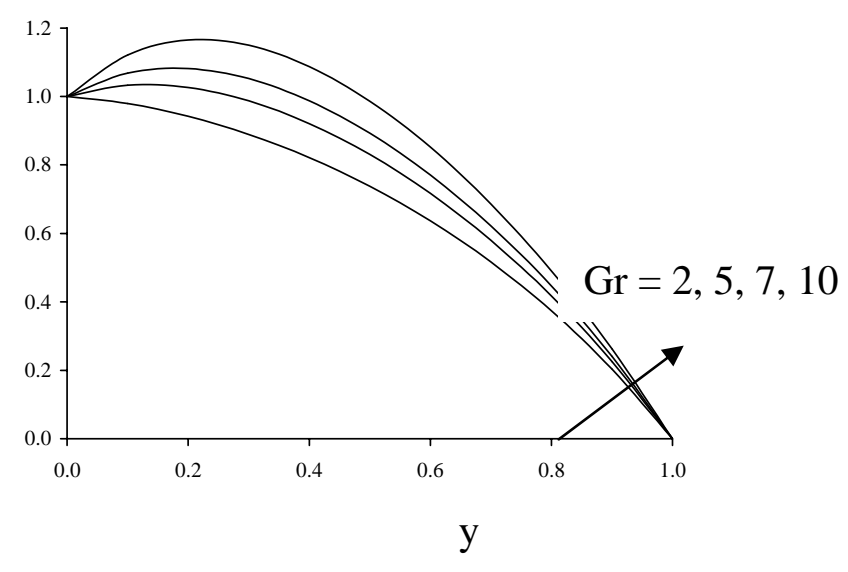

Fig 1: Velocity profiles as a function of $y$ for variations in Grashof number Grashof number

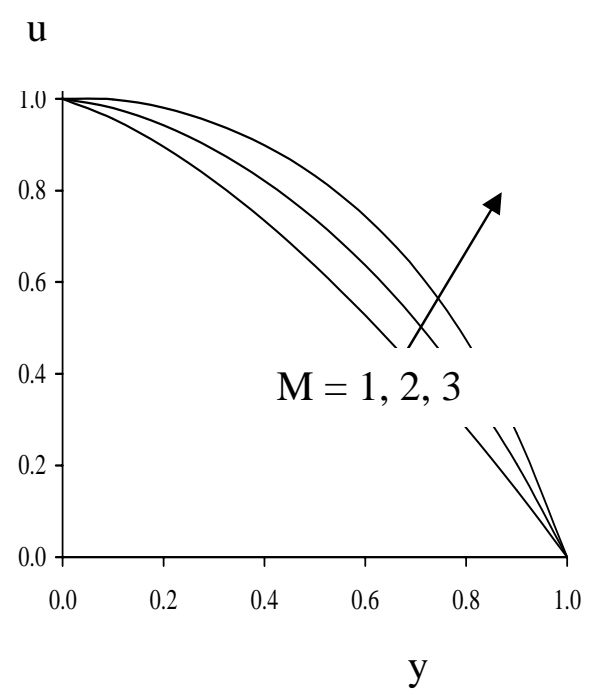

Fig 2: Velocity profiles as a function of $y$ for variations in Magnetic field 
u

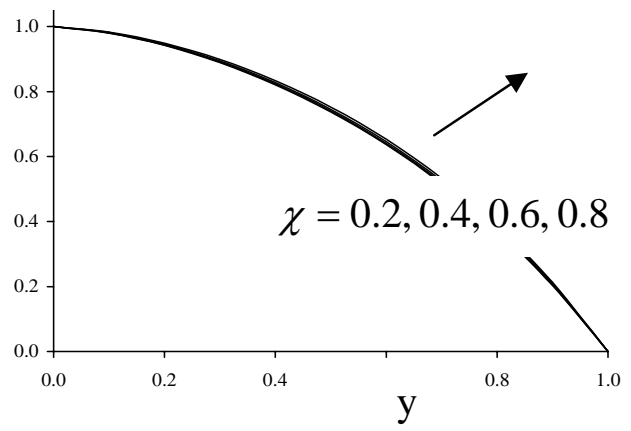

Fig 3: Velocity profiles as a function of $y$ for variations in porosity parameter.

$\mathrm{u}$

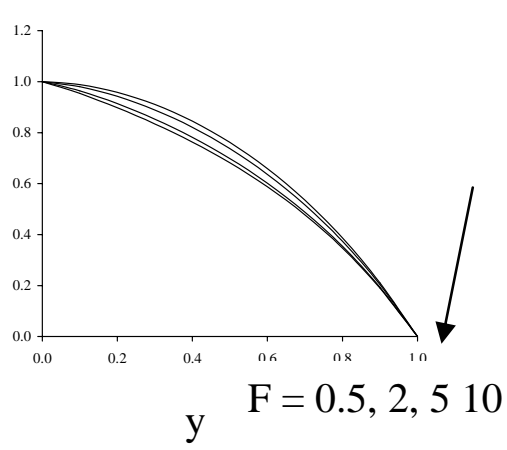

Figure 4: Velocity profiles as a function of $y$ for variations in radiation parameter
Further, it is observed in Figure 5 that the temperature profile decreased with increase in radiation. The magnitude of Nusselt number for various values of the material parameters are given in Table 4. It is seen that increase in radiation is associated with increase in the rate of heat transfer.

Table 4: Rate of heat transfer, $\mathrm{Nu}$ for variation of radiation parameter.

\begin{tabular}{|l|l|}
\hline $\mathrm{F}$ & $|N u|$ \\
\hline 0.5 & 1.10024 \\
1.0 & 1.32751 \\
1.5 & 1.66653 \\
2.0 & 2.07957 \\
2.5 & 2.53619 \\
\hline
\end{tabular}

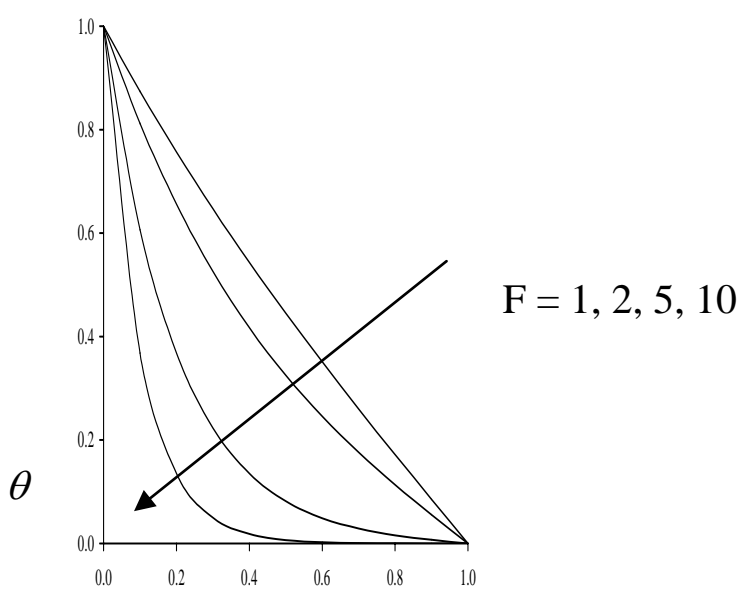

y

Fig 5: Temperature profiles as a function of $y$ for variations in radiation parameter

\section{CONCLUSION}

The problem of MHD free convection and oscillatory flow of an optically thin fluid bounded by two parallel walls under the influence of an external imposed transverse magnetic field in a porous medium has been examined. The effects of radiation, porosity, magnetic field and Grashof number on the velocity and temperature profiles as well as on the skin friction and rate of heat transfer have been 
investigated. The results indicate that these material parameters have significant influences on the velocity, temperature, skin friction and the rate of heat transfer. Generally, our results show that

- $\quad$ the fluid velocity increased with increase in the magnetic field, $M$, Grashof number, $G r$, and the porosity, $\chi$ parameters

- the fluid velocity is decreased with increase in the radiation parameter, $F$

- $\quad$ the skin friction decreased with increases in magnetic and porosity parameters for $G r=2$ and increased for $G r=5$

- $\quad$ the skin friction increased with increases in radiation for $G r=2$ and decreased for $G r=5$

- $\quad$ increase in radiation is associated with increase in the rate of heat transfer.

\section{REFERENCES}

Alagoa, K.D., Tay, G. and Abbey, T.M (1999). Radiative and free convective effects of a MHD flow through a porous medium between infinite parallel plates with time- dependent suction, Astrophysics and Space Science 260:455 - 468

Attia, H.A., Sayed-Ahmed, M.E (2004), Hall effect on unsteady MHD Couette flow and heat transfer of a Bingham fluid with suction and injection, Applied Mathematical Modelling 28: 1027 - 1045.

Bestman, A.R., and Adjepong, S. K (1988), Unsteady hydromagnetic free-convection flow with radiative heat transfer in a rotating fluid, Astrophysics and Space Science, 143:73- 80.

Choi, C.K., Chung, T.J., Kim, M.C (2004), Buoyancy effects in plane Couette flow heated uniformly from below between two parallel walls with constant heat flux, Int. J. Heat Mass Transfer, 47:2629 - 2636.

Cogley, A. C. L., Vincenti, W. G., Gilles, E. S (1968), Differential approximation for radiative heat transfer in a non grey gas near equilibrium, Am. Inst. Aeronat. Astronaut. J 6: $551-553$.

Ghoshdastidar, P.S (2004), Heat Transfer, Oxford University Press.

Israel-Cookey, C., Ogulu, A and Omubo-Pepple, V. B (2003), Influence of viscous dissipation and radiation on unsteady MHD free-convection flow past an infinite heated vertical plate in a porous medium with timedependent suction, International Journal of Heat and Mass transfer 46: 2305-2311.

Makinde, O. D., Osalusi, E (2006), MHD steady flow in a channel with slip at permeable boundaries, Romanian J. Physics 51(3-4): $319-328$.

Mebine, P (2007), Radiation effects on MHD Couette flow with heat transfer between two parallel plates, Global J. Pure Applied Mathematics, 3(2); 191 - 202.

Nield, D.A., Bejan, A (2006), Convection in Porous Media, Springer.

Wolfram, S (1991), Mathematica: A System of Doing Mathematics by Computers, Addison Wesley Publishing. 